\section{Perfil nutricional en estudiantes de nuevo ingreso de la Licenciatura en Nutrición de la Universidad Autónoma del Carmen.}

\section{Nutritional profile in new students of the nutrition bachelor's degree at Universidad Autonoma del Carmen.}

DOI: https://doi.org/10.19136/mhr.a2n3.1945

\author{
Angel Esteban Torres Zapata ${ }^{1^{*}}$ \\ Yasmin Escalante García ${ }^{1}$ \\ Javier Rivera Domínguez ${ }^{1}$ \\ Olga Chalim Solís Cardouwer ${ }^{1}$ \\ Claudia Elizabeth Guadarrama López ${ }^{1}$ \\ Juan Eduardo Moguel Ceballos 1 \\ 1Universidad Autónoma del Carmen
}

\section{Resumen}

El sobrepeso y obesidad son un problema de salud pública en México, el cual es abordada de forma multi e interdisciplinaria, por médicos, enfermeros, psicólogos, odontólogos, educadores físicos, fisioterapeutas y nutriólogos. Sin embargo, existen casos de profesionales sanitarios que manifiestan problemas de salud nutricional y que luchan contra su propio sobrepeso o la obesidad, y quizás sus pacientes los perciban de diferente manera con respecto a aquellos profesionales de la salud que se encuentren "esbelto".

Objetivo: Determinar el perfil nutricional a través de indicadores antropométricos de los estudiantes de nuevo ingreso de las generaciones 2014, 2015 y 2016.

Método: Estudio cuantitativo con un diseño no experimental transversal acerca del perfil nutricional de 139 estudiantes. El perfil antropométrico se registró en la cédula de evaluación del estado nutricio del Laboratorio de Evaluación del Estado Nutricio (LEEN) de la Facultad de Ciencias de la Salud de la UNACAR. Se tomaron medidas de peso, talla y circunferencia de la cintura y cadera por la responsable del Laboratorio de acuerdo a la norma estandarizada ISAK Nivel 1. Los datos fueron procesados con el paquete estadístico SPSS versión 24.0 para Windows. Se usó estadística descriptiva.

Resultados: Más de la mitad de los sujetos de estudios de nuevo ingreso de la Licenciatura en Nutrición de las generaciones 2014, 2015 y 2016 presentaron un estado nutricional incorrecto según los indicadores utilizados. Las mujeres de la cohorte generacional con mayor malnutrición fue la 2014 con una prevalencia combinada de sobrepeso y obesidad de $39.48 \%$ y en los varones la 2016 con una prevalencia combina del $50 \%$.

Conclusión: Se considera indispensable continuar con la evaluación del estado nutricio en la población estudiantil para identificar factores de riesgo y establecer diagnósticos certeros en etapas tempranas.

Palabras Clave: Perfil nutricional, obesidad, estudiantes universitarios. 


\begin{abstract}
Overweight and obesity are a public health issue in Mexico, which is addressed in a multi and interdisciplinary way, by doctors, nurses, psychologists, dentists, physical education professionals, physiotherapists and nutrition specialists. However, there are cases of healthrelated professionals that have nutritional issues and are fighting against their own overweight or even obesity, and perhaps are being seen in a different way by their patients when compared with other healthrelated professionals who are "slim".

Objective: The main goal of this research, was to determine, through anthropometrical indicators the nutritional profile of new students for the generations 2014, 2015, and 2016.

Method: A quantitative, transversal, non-experimental study was conducted on the nutritional profile of 139 students. The anthropometrical profile was recorded on a file kept at the Nutritional Status Evaluation Lab (NSEL) of the Health Science Division at the UNACAR. Weight, size, waist and hip diameter measures were taken by the laboratory assistant, observing Level 1 ISAK standards. Data was processed on SPSS 24.0 for windows. Descriptive statistical methods were used.

Results: More than half of the new student's population for the 2014, 2015, and 2016 generations, have an inadequate nutritional status. On 2014 female students had the higher percentage of incorrect nutritional status with a $39.48 \%$ combined presence of overweight and obesity, while in 2016 male students presented a $50 \%$ combined presence.

Conclusions: It is considered a necessity to keep doing research on the nutritional status of the student population to identify risk factors and to stablish accurate diagnosis on early stages.
\end{abstract}

Keywords: Nutritional profile, Obesity, University Students.
La salud como un derecho humano fundamental e indispensable para el ejercicio de los demás derechos humanos, está contemplada jurídicamente en el artículo 12 del Pacto Internacional de los Derechos Económicos, Sociales y Culturales, el cual establece: "El derecho al disfrute del más alto nivel posible de salud", ya que las condiciones de salud son, en gran medida, resultantes de las condiciones existentes para ejercer los derechos económicos, sociales y culturales (Comisión Nacional de los Derechos Humanos, 2012).

En México, durante el inicio del Siglo XXI, el índice de población con problemas de sobrepeso y obesidad ha incrementado hasta el punto de considerarse un problema de salud pública, de acuerdo a la Encuesta Nacional de Salud y Nutrición 2016 de medio camino (Secretaría de Salud, 2016), pues esta condición se considera como factor de riesgo desencadénate de diversas enfermedades crónicas, que afecta de igual manera a población adulta como infantil, ya que se ha identificado:

Tres de cada diez niños en edad escolar de 5 a 11 años de edad padecen sobrepeso u obesidad (prevalencia combinada de 33.2\%). Cuatro de cada diez adolescentes (12 a 19 años de edad) presenta sobrepeso u obesidad (prevalencia combinada de $36.3 \%$ ). Siete de cada diez adultos mayores de 20 años de edad presentan sobrepeso u obesidad (prevalencia combinada de 72.5\%). Esta condición impacta a la población colocándolos en situación de vulnerabilidad ante estereotipos negativos, los cuales generan prejuicios y discriminación social (Puhl, Gold, Luedicke, \& DePierre, 2013).

Esta situación de alteración del estado de salud es abordada de forma multi e interdisciplinaria por diferentes profesionales de la salud, como: médicos, enfermeros, psicólogos, odontólogos, educadores físicos, fisioterapeutas y nutriólogos. Siendo principalmente el nutriólogo, el profesional que durante su formación académica desarrolla las competencias profesionales para brindar cuidado nutricio a sujetos en diferentes condiciones: sanos, en riesgo o enfermos, tanto en lo individual como en grupos, en diferentes sectores de la sociedad; realizando funciones de gestión de servicios y programas de alimentación y nutrición; de formular, modificar y perfeccionar la calidad nutrimental y sanitaria de productos alimenticios. Pues el nutriólogo desarrolla las capacidades para aplicar, generar e integrar habilidades, actitudes y conocimientos, que permiten su desempeño en los campos profesionales 
(nutrición poblacional, clínica, tecnología alimentaria, servicios de alimentos y otros campos transversales) aplicando técnicas, métodos, y tecnologías propias de la nutrición y ciencias afines que fundamenta su ejercicio profesional en un marco ético y multidisciplinario para responder con calidad y compromiso a las necesidades sociales de alimentación y nutrición presentes y futuras (UNACAR, 2010 y AMMFEN, 2016). Por tal motivo, como parte de su ejercicio profesional los nutriólogos deben mantener un estado nutricional adecuado para dar credibilidad a la profesión.

Numerosos estudios documentan actitudes prejuiciosas de los médicos, las enfermeras y otros profesionales sanitarios hacia los pacientes obesos (Puhl, Gold, Luedicke, \& DePierre, 2013). Sin embargo, existen casos de profesionales sanitarios que manifiestan problemas de salud nutricional y que luchan contra su propio sobrepeso o la obesidad, y quizás sus pacientes los perciban de diferente manera con respecto a aquellos profesionales de la salud que se encuentren esbeltos. Por lo que es preocupante que estos estereotipos negativos estén presentes en el ámbito de la atención de la salud.

Desde el 2002, periodo que se apertura el programa educativo de Licenciatura en Nutrición en la Facultad de Ciencias de la Salud de la Universidad Autónoma del Carmen (UNACAR), se ha observado cualitativamente que un grupo considerable de estudiantes desde que ingresan hasta que egresan padecen de estas alteraciones del estado nutricio, aunando a esto, que algunos campos clínicos solicitan estudiantes que como requisito no presenten sobrepeso u obesidad por tener programas específicos para combatir este problema de salud. Es por esto que en el presente estudio se determinó el perfil nutricional a través de indicadores antropométricos de los estudiantes de nuevo ingreso de las generaciones 2014, 2015 y 2016 que permita diseñar estrategias y programas de intervención nutricional en los casos que se requieran y que al momento de egresar como Licenciados en Nutrición presenten una composición corporal congruente a su profesión.

\section{Método}

Participantes

Se realizó un estudio cuantitativo con un diseño no experimental transversal acerca del perfil nutricional de los estudiantes de nuevo ingreso del programa educativo de Licenciatura en Nutrición de la Facultad de Ciencias de la Salud de la UNACAR en Ciudad del Carmen, Campeche. La muestra fue de tipo no probabilístico, en el que se incluyeron a la totalidad de alumnos de primer ingreso de las generaciones 2014, 2015 y 2016.

\section{Instrumentos y mediciones}

El perfil antropométrico se registró en la cédula de evaluación del estado nutricio (FO-FCS-LEEN-01) del Laboratorio de Evaluación del Estado Nutricio (LEEN) de la Facultad de Ciencias de la Salud de la UNACAR. Todos y cada uno de los aspectos incluidos en el documento han sido validados por la academia de Nutrición.

Se tomaron medidas de peso, talla y circunferencia de la cintura y cadera por la responsable del Laboratorio de acuerdo a la norma estandarizada por International Society for the Advancement of Kinanthropometry (ISAK) Nivel 1. Todas las mediciones antropométricas fueron tomadas de acuerdo a los protocolos de ISAK. El peso se midió en kilogramos con una balanza de piso Tanita (modelo TBF 410GS), con capacidad máxima de $200 \mathrm{~kg}$ y mínima de $100 \mathrm{~g}$. La talla se midió con un estadiómetro de pared, rango de $0-220 \mathrm{~cm}$ con $1 \mathrm{~mm}$ de precisión. A partir del peso y la talla se calculó el índice de peso corporal (IMC=P/(T)2) y determinó talla baja como lo indica la NOM-008-SSA3-2010. La circunferencia de cintura y cadera se midieron por triplicado obteniéndose la media con una cinta métrica Lufkin W606PM con una capacidad de capacidad $200 \mathrm{~cm}$ y $1 \mathrm{~mm}$ de precisión. Con los datos de cintura y cadera se calculó el índice de cintura cadera $(I C C=C / C)$ (Marfell-Jones, Olds, Stewart \& Carter 2008 y Fernández et al., 2014).

Procedimiento de reclutamiento y recolección de datos

Para llevar a cabo el estudio primero se obtuvo la autorización del Coordinador de la Licenciatura en Nutrición y el consentimiento informado con nombre y firma de cada uno de los participantes. Se les programo para asistir al LEEN de la Facultad de Ciencias de la Salud de la UNACAR para obtener los datos antropométricos.

\section{Aspectos éticos}

El presente trabajo se apegó a los principios éticos nacionales dispuesto en la NOM-012-SSA3-2012 e internacionales para las investigaciones médicas en 
seres humanos (Declaración de Helsinki. Helsinki, 2000).

\section{Análisis estadístico}

Los resultados fueron procesados con el paquete estadístico SPSS versión 24.0 para Windows. Se usó estadística descriptiva (frecuencias, porcentajes para las variables categóricas y medidas de tendencia central y dispersión para las variables numéricas) para determinar el perfil nutricional de los participantes.

\section{Resultados}

Características generales de la población

La población de estudio se conformó por el total de estudiantes de primer ingreso $(\mathrm{N}=139)$ del programa educativo de Licenciatura en Nutrición de la UNACAR de tres cohortes generacionales consecutivos 2014, 2015 y 2016, de los cuales el $77 \%$ (107) fueron mujeres y el $23 \%$ (32) fueron varones. En la generación 2014 el $37 \%$ (52) de los estudiantes, de los cuales el $27 \%$ (38) mujeres y $10 \%$ (14) fueron varones; en la generación 2015 participaron $39 \%$ (54) estudiantes donde el $30 \%$ (42) son mujeres y el $9 \%$ (12) son varones; $y$, de la generación 2016 participaron las $24 \%$ (33) de estudiantes donde el 19 $\%$ (27) fueron mujeres y $4 \%$ (6) fueron varones. En la Tabla 1 se detalla las características de la población estudiada en relación a la edad, peso, talla, circunferencia de cintura y cadera

Perfil nutricional por antropometría

A todos los sujetos de estudios se les pregunto su edad y se tomó las mediciones de peso, talla, circunferencia de la cintura y cadera para determinar el porcentaje de talla (estatura) baja, talla (estatura) promedio, IMC (bajo peso, normopeso, sobrepeso y obesidad) como lo indica la NOM-008-SSA3-2010, e
ICC (riesgo muy bajo, bajo y alto) teniendo en cuenta que según la Organización Mundial de la Salud (OMS) los valores normales del índice cintura-cadera son de 0,80 en la mujer y 1 en el hombre, valores superiores indicarían obesidad abdominovisceral, lo cual se asocia a un riesgo cardiovascular aumentado, por generación y género. En la Tabla 2., se describe la comparación del perfil antropométrico en mujeres y en la Tabla 3., en varones de las distintas generaciones.

\section{Discusión}

La obesidad es un problema de salud pública el cual incrementa la mortalidad y morbilidad por la relación con enfermedades que afectan a la mayoría de los sistemas del organismo. En México la prevalencia de exceso de peso está en aumento de forma alarmante, en la población masculina adulta el sobrepeso y obesidad aumentó en zonas rurales (de $61.1 \%$ en 2012 a $67.5 \%$ en 2016) mientras que se estabilizó en zonas urbanas, en las que se mantiene en un nivel elevado (69.9\%) (Secretaria de Salud, 2016).

Diversos autores han destacado que la población universitaria es un grupo especialmente vulnerable desde el punto de vista de la salud nutricional (Pi, 2015) y un estudiante de la Licenciatura en Nutrición no es la excepción, ya que al ingreso del joven a estudiar esta carrera solo se evalúa el perfil de ingreso que describe los aspectos de la formación que debe poseer el aspirante, como son conocimientos (química, biología, bioquímica, etc.), habilidades (Comunicar, manejar información en forma organizada, etc.) y actitudes (responsabilidad, Interés de servicio hacia la comunidad), pero en muy raras ocasiones se evalúa el estado nutricio y se le da un seguimiento hasta el momento de egresar. Cabe aclarar que determinar el perfil nutricional de los

Tabla 1.- Características promedio de los sujetos de estudio

\begin{tabular}{|c|c|c|c|c|c|c|c|c|c|c|c|c|}
\hline \multirow[t]{2}{*}{ Parámetros } & \multicolumn{4}{|c|}{ Total $(n=139)$} & \multicolumn{4}{|c|}{ Femenino $(n=107)$} & \multicolumn{4}{|c|}{ Masculino $(n=32)$} \\
\hline & $\bar{x}$ & $\mathrm{DE}$ & V.M & V. $\mathrm{m}$ & $\bar{x}$ & $\mathrm{DE}$ & V.M & V. $m$ & $\bar{x}$ & $\mathrm{DE}$ & V.M & V. $m$ \\
\hline Edad (años) & 21.96 & 4.29 & 49.0 & 19.0 & 22.05 & 4.76 & 49.0 & 19.0 & 21.65 & 2.25 & 31.0 & 19.0 \\
\hline Peso (kg) & 62.81 & 14.43 & 104.5 & 41.5 & 59.96 & 13.19 & 104.5 & 41.5 & 72.32 & 14.34 & 101.6 & 48.9 \\
\hline Talla (cm) & 159.92 & 8.07 & 180.0 & 140.0 & 157.35 & 6.47 & 173.0 & 140.0 & 168.46 & 6.90 & 180.0 & 150 \\
\hline Cintura (cm) & 77.11 & 10.89 & 112.0 & 57.0 & 75.59 & 10.00 & 107.0 & 57.0 & 82.58 & 12.16 & 112.0 & 65.0 \\
\hline Cadera $(\mathrm{cm})$ & 96.72 & 10.52 & 122.8 & 56.8 & 97.12 & 10.53 & 122.8 & 56.8 & 95.31 & 10.36 & 119.0 & 80.0 \\
\hline
\end{tabular}

V. M: Valor máximo, V. m: Valor mínimo 
Tabla 2. Comparación del perfil antropométrico en mujeres generacional.

\begin{tabular}{|c|c|c|c|c|c|c|c|c|c|c|c|c|}
\hline \multirow{3}{*}{ Indicadores } & \multicolumn{12}{|c|}{ Femenino } \\
\hline & \multicolumn{4}{|c|}{ Generación $2014(n=38)$} & \multicolumn{4}{|c|}{ Generación $2015(n=42)$} & \multicolumn{4}{|c|}{ Generación $2016(n=27)$} \\
\hline & $f$ & $\%$ & $\bar{x}$ & $\mathrm{DE}$ & $f$ & $\%$ & $\bar{x}$ & $\mathrm{DE}$ & $f$ & $\%$ & $\bar{x}$ & $\mathrm{DE}$ \\
\hline Talla baja & 3 & 7.89 & 147.73 & 0.900 & 3 & 7.14 & 142.36 & 1.670 & 6 & 22.22 & 148.18 & 1.34 \\
\hline Talla promedio & 35 & 92.11 & 159.65 & 5.510 & 39 & 92.86 & 158.50 & 5.570 & 21 & 77.78 & 158.00 & 4.45 \\
\hline IMC bajo peso & 3 & 7.89 & 16.40 & 0.160 & 1 & 2.38 & 15.60 & 0 & 2 & 6.06 & 16.80 & 0.20 \\
\hline IMC normopeso & 20 & 52.63 & 20.91 & 1.470 & 25 & 59.52 & 21.42 & 2.010 & 15 & 45.45 & 22.20 & 1.87 \\
\hline IMC sobrepeso & 10 & 26.32 & 26.53 & 1.130 & 13 & 30.95 & 27.56 & 1.450 & 6 & 18.18 & 26.92 & 1.69 \\
\hline IMC obesidad & 5 & 13.16 & 29.86 & 3.730 & 3 & 7.14 & 34.43 & 2.470 & 4 & 12.12 & 30.92 & 5.35 \\
\hline ICC riesgo muy bajo & 26 & 68.42 & 0.74 & 0.028 & 30 & 71.43 & 0.75 & 0.028 & 23 & 69.70 & 0.74 & 0.03 \\
\hline ICC riesgo bajo & 9 & 23.68 & 0.82 & 0.014 & 8 & 19.05 & 0.81 & 0.010 & 4 & 12.12 & 0.81 & 0.02 \\
\hline ICC riesgo alto & 3 & 7.89 & 0.87 & 0.010 & 4 & 9.52 & 0.87 & 0.010 & 0 & 0 & 0 & 0 \\
\hline
\end{tabular}

Tabla 3. Comparación del perfil antropométrico en varones generacional.

\begin{tabular}{|c|c|c|c|c|c|c|c|c|c|c|c|c|}
\hline \multirow{3}{*}{ Indicadores } & \multicolumn{12}{|c|}{ Masculino } \\
\hline & \multicolumn{4}{|c|}{ Generación 2014 (n=14) } & \multicolumn{4}{|c|}{ Generación 2015 (n=12) } & \multicolumn{4}{|c|}{ Generación 2016 (n=6) } \\
\hline & $f$ & $\%$ & $\bar{x}$ & $\mathrm{DE}$ & f & $\%$ & $\bar{x}$ & $\mathrm{DE}$ & $f$ & $\%$ & $\bar{x}$ & $\mathrm{DE}$ \\
\hline Talla baja & 2 & 14.29 & 151.00 & 1.00 & 0 & 0 & 0 & 0 & 0 & 0 & 0 & 0 \\
\hline Talla promedio & 12 & 85.71 & 168.29 & 5.81 & 12 & 100.00 & 170.60 & 5.980 & 6 & 100.00 & 167.40 & 6.100 \\
\hline IMC bajo peso & 2 & 14.29 & 17.05 & 0.05 & 0 & 0 & 0 & 0 & 0 & 0 & 0 & 0 \\
\hline IMC normopeso & 6 & 42.86 & 22.21 & 1.62 & 7 & 58.33 & 23.14 & 1.950 & 3 & 50.00 & 20.60 & 0.400 \\
\hline IMC sobrepeso & 4 & 28.57 & 26.90 & 1.37 & 3 & 25.00 & 28.33 & 1.240 & 2 & 33.33 & 26.55 & 0.450 \\
\hline IMC obesidad & 2 & 14.29 & 31.05 & 0.85 & 2 & 16.67 & 33.50 & 2.500 & 1 & 16.67 & 31.90 & 0 \\
\hline ICC riesgo muy bajo & 14 & 100.00 & 0.82 & 0.04 & 9 & 75.00 & 0.83 & 0.048 & 6 & 100.00 & 0.86 & 0.048 \\
\hline ICC riesgo bajo & 0 & 0 & 0 & 0 & 2 & 16.67 & 1.00 & 0 & 0 & 0 & 0 & 0 \\
\hline ICC riesgo alto & 0 & 0 & 0 & 0 & 1 & 8.33 & 1.20 & 0 & 0 & 0 & 0 & 0 \\
\hline
\end{tabular}

estudiantes, no debe ser un indicador para ser aceptado en el programa educativo.

En este estudio se determinó el perfil nutricional del estudiante de nuevo ingreso al programa educativo de Licenciatura en Nutrición desde el 2014 hasta el 2016 para generar estrategias y programas de intervención desde los primeros años de su profesión, donde se observó de manera general que el $55.40 \%$ presenta un estado nutricio inadecuado. La información que sostiene lo anterior se observa en lo siguiente:

El $10.07 \%$ (12 mujeres y 2 varones) de los sujetos de estudio presentan talla baja y $89.93 \%$ (95 mujeres y 30 varones) presentaron una talla promedio según la NOM-008-SSA3-2010. Siendo las mujeres en donde se refleja más este problema y al comparar con los datos del ENSANUT 2016 medio camino la prevalencia es menor $16.83 \%$.

De igual forma se determinó que el $45.32 \%$ tuvieron estados de inadecuada nutrición tanto por alteraciones de acuerdo con el IMC. Los puntos de corte de la norma oficial mexicana para el tratamiento integral del sobrepeso y la obesidad, se considera que un IMC <18.5 es indicativo de bajo peso 0 desnutrición; valor que se encontró en un $5.76 \%$ (6 mujeres y 2 hombres). Al comparar estos datos con el estudio realizado por Gallardo Wong y Abad Eslava en estudiantes universitarios de la Escuela de Dietética y Nutrición del ISSSTE, se observó una menor prevalencia de desnutrición con respecto a la población de estudio ( $7.5 \%$ ). Un IMC entre 18.5 y 25 es indicador de que el individuo está en un peso promedio ("normal"); valor que se encontró en un $54.68 \%$ (60 mujeres y 16 hombres), un $14.32 \%$ menor a lo reportado por Vargas y Becerra 2016 en un estudio sobre el estado nutricional y consumo de alimentos de estudiantes universitarios admitidos a nutrición y dietética en la Universidad Nacional De Colombia pero al comparar estos datos con el estudio realizado por Gallardo Wong y Abad Eslava en estudiantes universitarios de la Escuela de Dietética y Nutrición del ISSSTE, se observó un 11.48 \% más 
estudiantes con un IMC promedio con respecto a la población de estudio (47.2\%) y se encontró por arriba de la media nacional al compararlo por grupo etario de 20 a 29 años del ENSANUT 2016 Medio camino. EI IMC que indica sobrepeso se encontró en un 27.34 $\%$ (19 mujeres y 9 hombres), menor que lo reportado por Antonella Pi et al., 2015 (50 \%) y lo reportado el ENSANUT 2016 medio camino, pero teniendo un $12.24 \%$ más que lo reportado por Gallardo Wong y Abad Eslava. El IMC indicador de obesidad se determinó en un $12.23 \%$ (12 mujeres y 5 varones) ligeramente $(0.93 \%)$ más alto que en los estudiantes de nutrición de la Escuela de Dietética y Nutrición del ISSSTE, pero por debajo de la tendencia nacional. Otro parámetro que aporta información del estado nutricio, algo menos utilizado, es el Índice CinturaCadera (ICC), que además de indicar la tendencia 0 predisposición personal a acumular grasa, incide en la probabilidad de padecer enfermedades cardíacas, diabetes o problemas de tensión arterial. La OMS indica puntos de cortes tanto para hombres y mujeres, que son los que se utilizaron para este estudio donde se determinó que el $77.70 \%$ (79 mujeres y 29 varones) de los sujetos de estudio presentaban riesgo muy bajo. El $16.55 \%$ (21 mujeres y 2 varones) presentaban riesgo bajo y solo el $5.76 \%$ (7 mujeres y un hombre) presentaban un riesgo alto, estos resultados son muy parecido a lo reportado por otros autores tales como Hidalgo y Hidalgo 2014; Corvos, Corvos, y Armando, 2014.

Las mujeres de la cohorte generacional que se vio más afectado en relación al perfil nutricional fue la 2014 con una prevalencia combinada de sobrepeso y obesidad de $39.48 \%, 1.39 \%$ más que en la generación 2015 y un 9.28 \% que la generación 2016 . En el caso de los varones, se observó que la generación más afecta fue la 2016 con una prevalencia combina del $50 \%, 7.14 \%$ más que la cohorte generacional 2014 y $8.33 \%$ más que la 2015 .

\section{Conclusiones}

El sobrepeso y la obesidad en estudiantes y profesionales de la salud, especialmente de la nutrición los hacen vulnerables a estereotipos negativos, estos estereotipos generan prejuicios y discriminación a la hora de ejercer profesionalmente, por tal motivo es importante tener como un requisito la evaluación nutricional en todos los estudiantes del área de salud. En este estudio se concluye que un poco más de la mitad de los sujetos de estudio (alumnos) de nuevo ingreso de la Licenciatura en
Nutrición de las generaciones 2014, 2015 y 2016 presentaron un estado nutricio incorrecto según los indicadores utilizados. Se considera indispensable continuar con la evaluación del estado nutricio en la población estudiantil para identificar factores de riesgo y establecer diagnósticos certeros en etapas tempranas, con el propósito de promover estrategias preventivas y correctivas que eviten el desarrollo de enfermedades crónicas degenerativas 0 complicaciones, debido a que en los próximos años constituirán a la población productiva y económicamente activa de nuestro país.

\section{Declaración de conflicto de interés}

No se reporta conflicto alguno.

\section{Referencias}

AMMFEN (Asociación Mexicana de Miembros y Facultades de Nutrición). (2016). Campos profesionales. Recuperado de http://ammfen.mx/campos-profesionales.aspx

Antonella Pi, R., Daniela Vidal, P., Romina Brassesco, B., \& Rosana Aballay, L. (2015). Estado nutricional en estudiantes universitarios: su relación con el número de ingestas alimentarias diarias y el consumo de macronutrientes. Nutrición Hospitalaria, 31(1), 1748-1756. Recuperado de DOI:10.3305/nh.2015.31.4.8399

Carías, D., Cioccia, A. M., Gutiérrez, M., Hevia, P., \& Pérez, A. (2009). Indicadores bioquímicos del estado nutricional en adolescentes preuniversitarios de Caracas. Anales Venezolanos de Nutrición, 22(1), 12-19. Recuperado de http://web.b.ebscohost.com/ehost/pdfviewer/pdfvie wer?sid=a00f410a-4769-4a49-80b2-

9281 c 2 ba4347\%40sessionmgr 101 \&vid $=1$ \&hid $=12$ 9

Comisión Nacional de los Derechos Humanos. México. (2012). Colección del Sistema Universal de Protección de los Derechos Humanos. El Pacto Internacional de Derechos Económicos, Sociales y Culturales. Recuperado de http://stj.col.gob.mx/dh/html/biblioteca/descargable s/pdf/3/i/4.pdf

Corvos Hidalgo, C A; Corvos Hidalgo, A; (2014). Índices antropométricos como predictores de riesgo cardiovascular en universitarios. Multiciencias, 14() 196-202. Recuperado de 
http://www.redalyc.org/articulo.oa?id=9043260101 1

Corvos, C., Corvos, A., \& Armando, S. (2014). Índices antropométricos y salud en estudiantes de ingeniería de la Universidad de Carabobo. Nutrición Clínica y Dietética Hospitalaria, 34(2), 45-51. Recuperado de DOI: 10.12873/342carabobocorvos Fernández Cabrera, Jacinta, Aranda Medina, Emilio, Córdoba Ramos, María de Guía, Hernández León, Alejandro, Rodríguez Bernabé, José Antonio, \& Pérez-Nevado, Francisco. (2014). Evaluación del estado nutricional de estudiantes adolescentes de Extremadura basado en medidas antropométricas. Nutrición Hospitalaria, 29(3), 665-673. Recuperado de DOl.org/10.3305/NH.2014.29.3.7070

Figueroa López, C G; Ramos del Rio, B; (2006). Factores de riesgo de la hipertensión arterial y la salud cardiovascular en estudiantes universitarios. Anales de Psicología, 22() 169-174. Recuperado de http://www.redalyc.org/articulo.oa?id=16722201

Gallardo Wong, I; Arreguín Daza, T; Bernal Huerta, K; (2012). Correlación de la composición corporal por plicometría y bioimpedancia en estudiantes de nutrición. Revista de Especialidades MédicoQuirúrgicas, 17() 15-19. Recuperado de http://www.redalyc.org/articulo.oa?id=4732326000 4

Gallardo, I., \& Buen, L. L. (2011). Mala nutrición en estudiantes universitarios de la Escuela de Dietética y Nutrición del ISSSTE. Rev. Med UV, 1, 7-1. Recuperado de https://www.uv.mx/rm/num_anteriores/revmedica_ vol11_num1/articulos/mala.pdf

Instituto Nacional de Salud Pública. Encuesta Nacional de Salud y Nutrición de Medio Camino 2016 Informe Final de Resultados. Recuperado de http://oment.uanl.mx/wpcontent/uploads/2016/12/ensanut_mc_2016310oct.pdf

Marfell-Jones, M., Olds, T., Stewart, A., \& Carter, L. (2008). Estándares internacionales para la evaluación antropométrica (3rd ed.). South Africa: La sociedad internacional para el avance de la cineantropometría (ISACK).

Norma Oficial Mexicana NOM-012-SSA3-2012, Que establece los criterios para la ejecución de proyectos de investigación para la salud en seres humanos. Diario oficial de la federación. Recuperado http://dof gob mx/nota detalle php?codigo $=528414$ 8\&fecha $=04 / 01 / 2013$
Pi, R A; Vidal, P D; Brassesco, B R; Viola, L; Aballay, L R; (2015). Estado nutricional en estudiantes universitarios: su relación con el número de ingestas alimentarias diarias y el consumo de macronutrientes. Nutrición Hospitalaria, 31() 1748$1756 . \quad$ Recuperado de http://4www.redalyc.org/articulo.oa?id=309238513 040

Puhl, R. M., Gold, J. A., Luedicke, J., \& DePierre, J. A. (2013). The effect of physicians' body weight on patient attitudes: implications for physician selection, trust and adherence to medical advice. International Journal of Obesity, 37, 1415-1421. Recuperado de doi:10.1038/ijo.2013.33

Secretaría de Salud. (2016). Encuesta Nacional de Salud y Nutrición de Medio Camino, 2016 (ENSANUT MC, 2016). Retrieved from http://www.gob.mx/cms/uploads/attachment/file/20 9093/ENSANUT.pdf

UNACAR (Universidad Autónoma del Carmen). (2010). Facultad de Ciencias de la Salud. Licenciatura en Nutrición. Recuperado de http://www.unacar.mx/contenido/oferta_educativa_ 2014/LICENCIATURA_SALUD/LNUTRICION/misi on.html

Vargas, M. E., \& Becerra, F. (2016). Estado nutricional y consumo de alimentos de estudiantes universitarios admitidos a nutrición y dietética en la Universidad Nacional De Colombia. Revista de Salud Pública, 17(5), 762-775. Recuperado de doi:10.15446/rsap. v17n5.43570

Vargas-Zárate, M; Becerra-Bulla, F; Prieto-Suárez, E; (2010). Evaluación de la ingesta dietética en estudiantes universitarios. Bogotá, Colombia. Revista de Salud Pública, 12() 116-125. Recuperado de http://www.redalyc. org/articulo.oa?id=4221901001 1

Fecha de recepción: 2-abr-2017 Fecha de aceptación: 5-jul-2017 